\title{
Neurological Aspects of the COVID-19 Pandemic
}

\author{
Alexandra Zirra ${ }^{1}$ and Cris S Constantinescu² \\ 1. Renal Medicine Unit, Barking, Havering and Redbridge University Hospitals NHS Trust, London, UK; 2. Division of Clinical Neuroscience, \\ Section of Clinical Neurology, University of Nottingham, Nottingham University Hospitals NHS Trust, Nottingham, UK
}

DOI: https://doi.org/10.17925/ENR.2020.15.1.13

\begin{abstract}
$\mathrm{T}$ he recent outbreak of the novel coronavirus, SARS-COV-2 - which causes COVID-19 - has rapidly reached pandemic proportions, causing widespread morbidity and mortality. It has had a major impact on society in general, and in medicine and health in particular. Although primarily a potentially fatal respiratory pathogen, SARS-CoV-2, like other coronaviruses, can be neurotropic. Its receptor, angiotensin-converting enzyme 2 (ACE2), is expressed in endothelial cells in the brain and can be induced in neural cells. COVID-19 is therefore of high relevance to neurological disease. Indeed, various neurological manifestations are increasingly being reported. On the other hand, COVID-19 has a major impact on people with certain neurological diseases. Patients with neuroimmune disorders such as multiple sclerosis or myasthenia gravis who are on immunomodulatory or immunosuppressive treatments may be more susceptible to infection or have a more severe disease due to a blunted immune response. Patients with many neurological disorders may be at risk of severe COVID-19 infection, due to immobility, frailty, disability, diminished respiratory capacity or cognitive dysfunction. Disease-specific patient organisations and neurological societies have been prompt in providing advice and generating guidelines for various neurological conditions. Local, national and international registers are being created and will be essential in monitoring the disease and its outcomes. New knowledge about COVID-19, including issues of relevance to neurology, is generated daily. This review presents some succinct background information on coronaviruses and its neuro-invasive potential, the current knowledge of neurological manifestations, and the impact of COVID-19 on individuals with neurological disease. Some disease-specific recommendations are briefly listed or referenced.
\end{abstract}

\section{Keywords}

COVID-19, coronavirus, neurotropism, central nervous system (CNS), stroke, multiple sclerosis, myasthenia gravis

Disclosures: Alexandra Zirra and Cris S Constantinescu have no financial or non-financial relationships or activities to declare in relation to this article.

Review Process: Double-blind peer review. Compliance with Ethics: This study involves a review of the literature and did not involve any studies with human or animal subjects performed by either of the authors.

Authorship: The named authors meet the International Committee of Medical Journal Editors (ICMJE) criteria

for authorship of this manuscript, take responsibility

for the integrity of the work as a whole, and have given final approval for the version to be published.

Access: This article is freely accessible at

touchNEUROLOGY.com (C) Touch Medical Media 2020

Received: 18 April 2020

Accepted: 9 June 2020

Published Online: 4 September 2020

Citation: European Neurological Review. 2020;15(1):13-7

Corresponding Author: Cris S constantinescu,

Division of Clinical Neuroscience, Section of

Clinical Neurology, University of Nottingham,

Queen's Medical Centre, Nottingham NG7 2UH,

UK. E: cris.constantinescu@nottingham.ac.uk

Support: No funding was received in

the publication of this article.
In the past 4 months, a novel coronavirus originating in Wuhan, China, SARS-CoV-2 (severe acute respiratory syndrome coronavirus 2), has affected more than 200 countries. As of mid-June 2020, there are over 7 million confirmed cases and more than 425,888 deaths worldwide caused by the novel coronavirus, although the numbers are likely to be higher because of variability in diagnostic testing rates and in attribution of deaths to the infection. ${ }^{1}$

\section{Coronaviruses}

SARS-CoV-2 is a positive-sense RNA Betacoronavirus from the Coronaviridae family. Coronaviruses are usually found in mammalian (Alpha-, Beta-, Deltacoronavirus) or avian (Gamma- and Deltacoronavirus) reservoirs. Some human endemic coronaviruses have been known since the 1960s and cause up to 20\% of upper respiratory tract infections (Alphacoronavirus: NL63, 29E, and Betacoronavirus: OC43, HKU1). ${ }^{2}$ However, other Betacoronaviruses have caused human pandemics with high mortality, such as SARS-COV in 2002-2003 originating in the Guangdong province in China, with a case-fatality rate (CFR) of 10\%, and MERS-CoV in 2012 in the Middle East (CFR 35\%).,3.4

The structure of coronaviruses is conserved between coronavirus genera. It comprises non-structural proteins (polyprotein 1a and $1 \mathrm{~b}$ ) and structural proteins (spike [S], envelope [E], membrane [M] and nucleocapsid [N]). The structural proteins are interspersed with open-reading frames (ORFS). ${ }^{4}$ SARS-CoV-2 was genotyped in January 2020 and has shown a high sequence homology with SARS-COV and MERS-COV. Nevertheless, phylogenetic proximity differs depending on sequence analysed. Indeed, when looking at ORF1a, protein E and M, the closest homology is with bat SL-COVZC45 and bat SL-CoVZXC21. On the contrary, when analysing S protein - responsible for virulence in Coronaviridae - SARS-COV-2 shows greatest homology with SARS-CoV and SARS-related CoVs (73.8-76.9\% amino acid identity). ${ }^{5}$

SARS-COV and related coronaviruses have a receptor-binding domain that uses the angiotensin-converting enzyme 2 (ACE2) protein as a receptor for cell entry. ${ }^{6}$ The ACE2 receptor is expressed in lung alveolar type 2 cells, epithelial cells from the lung, gastrointestinal tract, urinary bladder, proximal tubule cells and myocardial cells. ${ }^{7.8}$ There is evidence for the expression of ACE2 in the brain. ACE2 mRNA is expressed in cortex, striatum, hypothalamus and brainstem. Its abundance on endothelial cells, including those of the brain vessels, can explain the vascular (including neuro-vascular) complications of COVID-19.8-11 SARS-COV-2 engagement of ACE2 is more effective and extensive than that of other coronaviruses. ${ }^{12}$ As ACE2 is counterbalancing the vasoactive effects of angiotensin II, and SARS-COV-2 invasion 
destroys the ACE2 receptors, the pathogenesis of COVID-19 may be related to unopposed effects of angiotensin $\|_{1}{ }^{13}$ which explains some of the vascular complications and the worse prognosis in individuals with cardiovascular disorders. As with other coronaviruses, alternative receptors may be implicated, in particular $\mathrm{CD} 147,{ }^{14}$ which is also expressed in the central nervous system (CNS) and up-regulated by injury or inflammation. ${ }^{15}$

\section{Neurotropism of coronaviruses}

Coronaviruses have long been known to display neurotropism in animals and humans. The role of the coronavirus mouse hepatitis virus (MHV)-induced demyelination as a well-established model for multiple sclerosis (MS) has been extensively investigated. ${ }^{16}$ The neurovirulence of neurotropic MHV strains has been shown to be associated with their ability to induce inflammatory cytokines in neuroglial cells, which is relevant in view of the role of the cytokine storm in the pathogenesis of COVID-19. ${ }^{17}$ These early studies also provided the first hints to the routes and mechanisms of neuroinvasiveness of coronaviruses, i.e., via the airways, olfactory bulbs, and possibly trans-synaptic transmission, all of which are likely relevant to COVID-19.18

Not only are some coronaviruses neurotropic, but they may also establish persistence within the $\mathrm{CNS}_{1}{ }^{19}$ even in patients with other neurological disorders, like MS. ${ }^{20}$ Indeed SARS-CoV has been shown to enter the CNS via the cribriform plate or a damaged blood-brain barrier, neurotropism mediated by ACE2 protein. Moreover, SARS-COV was found in the cerebrospinal fluid (CSF). ${ }^{8}$ Given the high homology of the spike protein with SARS-CoV, it is possible that SARS-COV-2 is found in the CNS with a similar mechanism. However, it is worth noting that so far, even with neurological involvement, there is only one case report where SARS-COV-2 was identified in the CSF by molecular diagnosis. ${ }^{21}$ If the invasion occurs via the blood-brain barrier, then there is a theoretical risk of bleeding and haemorrhagic stroke, as well as local inflammation. ${ }^{8}$

SARS and MERS infections have previously been recognised to have neurological manifestations and they were found to have prominent brainstem involvement, likely via trans-synaptic, haematogenic or lymphatic routes. It has been suggested that the lack of good respiratory control seen in patients with COVID-19 with severe disease could be the cause of or contribute to respiratory distress. ${ }^{22}$ It was therefore not unexpected that reports of neurological involvement emerged soon after the breakout of the COVID-19 pandemic.

In view of the above considerations, and with the knowledge of the distribution of the ACE2 receptor, it was hypothesised early in the pandemic that COVID-19 can affect the brain and is likely to involve the brainstem structures.

\section{Clinical aspects of SARS-CoV-2}

COVID-19 most commonly presents in males on average 54 years old, ${ }^{23}$ after an incubation period of 5 days (range, 1-12 days) with dry cough, fever and myalgia. ${ }^{24,25}$ These symptoms can remain mild or can progress to shortness of breath and need for oxygen therapy (moderate), non-invasive ventilation (severe) or invasive ventilation with multiorgan support (severe/critical). Other associated symptoms can be gastrointestinal (diarrhoea, nausea, loss of appetite), dermatological (ranging from maculo-papular and vesicular eruptions to urticaria to [pseudo]-chilblains to livedoid necrosis) ${ }^{26,27}$ and neurological (muscle weakness, dysgeusia/anosmia, headache, photophobia, and focal neurological finding).
SARS-COV-2 pneumonia is diagnosed based on lymphopaenia, high CRP, chest radiographs with interstitial pattern/bilateral alveolar infiltrates, computerised tomography of the chest showing consolidation and viral polymerase chain reaction (PCR) of the throat and nose swab. Other common laboratory findings are: low albumin, deranged liver function tests, high lactate dehydrogenase and high erythrocyte sedimentation rate. ${ }^{28}$

Poor prognostic factors have been identified as male sex, older age (>65 years), comorbidities (hypertension, diabetes, cardiovascular and cerebrovascular disease), smoking, lymphocyte count $<1 \times 10^{\circ} / \mathrm{L}$, high CRP (>100 mg/L), high ferritin, acute kidney injury, coagulopathy with thrombocytopaenia and raised D-dimers $(1.5 \mu \mathrm{g} / \mathrm{mL}){ }^{29-32}$ Treatment is supportive with paracetamol, aiming for a neutral fluid balance, antibiotic/ antiviral (oseltamivir) cover, oxygen therapy from nasal cannula to invasive ventilation or extracorporeal membrane oxygenation, ${ }^{33}$ forced expiratory techniques and placing the patient in prone position. ${ }^{34}$ Clinical studies of antivirals, ${ }^{22}$ antimalarials, ${ }^{35,36}$ steroids, ${ }^{37}$ immunotherapies ${ }^{37}$ and convalescent plasma ${ }^{38,39}$ are ongoing. Some of these study results are emerging. Although definitive conclusions for hydroxychloroquine and chloroquine studies await class I evidence, they do not appear to have a major positive effect. ${ }^{40}$ On the other hand, remdesivir, an antiviral developed for Ebola, showed promising results in the interim analysis of an ongoing clinical trial. ${ }^{36,41}$ Furthermore, a larger trial of the antiviral together with either placebo or baricitinib (a Jak1/Jak2 kinase inhibitor that is aimed at reducing the cytokine induction) has been initiated at the National Institutes of Health.

\section{Neurological manifestations of COVID-19}

Given the neurotropism of coronaviruses, it was not unexpected that reports of neurological involvement emerged soon after the outbreak of the COVID-19 pandemic. In the series of 214 patients hospitalised with the infection in Wuhan, $36.4 \%$ had neurological manifestations. ${ }^{42}$ These included dizziness, headaches, anosmia/ageusia, seizures, stroke, altered consciousness and ataxia. This represents a list of symptoms roughly in the order of their frequency, and, as the publication was not focused on neurological symptoms, there was no distinction between the non-specific effects of a severe infectious state and specific neurological effects potentially indicating direct neuropathology. Neurological involvement was seen in the more severely affected patients. The alteration in smell and taste seems to be widespread and can represent an early and specific marker of COVID-19. ${ }^{43}$

In a subsequent series of 68 consecutive patients admitted due to acute respiratory distress syndrome caused by COVID-19 to two intensive care units (ICU) in France, 58 had neurological abnormalities. Some of these were diffuse, non-specific and non-localising, such as fever, agitation, confusion and dysexecutive syndrome; whether they are attributed to direct viral effect, anaesthesia and sedation, or ICU/critical illness encephalopathy is uncertain. Some imaging and CSF findings were more specific and included leptomeningeal enhancement, perfusion abnormalities (bilateral frontotemporal hypoperfusion in all patients undergoing perfusion imaging), cerebral ischaemic strokes (discussed below), oligoclonal bands matched in CSF and blood, and increased immunoglobulin G in CSF. Of note, none of the seven patients tested had molecular evidence of virus in the CSF. ${ }^{44}$

Stroke has now become a recognised complication of COVID-19. Increasing evidence points to dysregulated coagulation in COVID pathology, including pulmonary manifestations which may be due to microthrombi. ${ }^{30,45}$ There is disseminated intravascular coagulation 
occurring more frequently than with SARS of other causes, which not only explains the presence of deranged laboratory findings such as D-dimers and abnormal coagulation tests, but also complications such as pulmonary emboli, some of the skin manifestations such as 'COVID fingers and toes' with chilblains, livedoid lesions and possible necrosis, and also ischaemic stroke. Thus, strokes in COVID-19 are explained in part by a hyperthrombotic state triggered by the virus. In addition, antiphospholipid antibodies, which are linked to thrombosis, have been detected in COVD-19-related thrombotic complications. ${ }^{46}$ Despite reported thrombocytopaenia, haemorrhages are not reported and the treatment of stroke in COVID-19 follows the same principles, with no contraindication for anticoagulation, thrombolysis or thrombectomy. ${ }^{47}$

The report in the radiology literature of COVID-19-associated acute necrotising haemorrhagic encephalopathy with typical imaging alerted clinicians to this possible rare complication which has been seen with other viruses. ${ }^{48}$ The patient tested positive for SARS-CoV-2 by PCR in nasopharyngeal swab, but the CFS could not be tested. Guillain-Barré syndrome has also been reported in the setting of COVID-19. ${ }^{49}$ As mentioned above, a case of COVID-19 meningo-encephalitis is to our knowledge, the only report of positive identification for virus by PCR in the CSF. ${ }^{21}$ There is no doubt that further reports and case series of neurological manifestations will be published and will shed further light on the clinical features of this condition.

\section{Neurological considerations in people recovering from COVID-19}

The creation of registers with documentation of proven or suspected cases and sharing of data between centres began in the early days of the pandemic. For example, the Association of British Neurologists provides both online guidance to patients and the opportunity for neurologists to register cases (www.theabn.org/page/COVID-19) Likewise, Italian neurologists have collected cases of MS, provided guidelines, and published their results; the register is still ongoing (https://musc-19.dibris.unige.it/). ${ }^{50}$ The follow-up of these people will be of great importance to provide insights into the characteristics of the virus and its interactions with the host, to address issues related to the natural or vaccine-induced antiviral immunity and to viral persistence, and to monitor for late complications. From the experience with other coronaviruses which can induce cross-reactive immune responses with neuroantigens, and with other viral infections such as influenza, awareness is needed of post-infectious immune-mediated neurological conditions, including acute disseminated encephalomyelitis, other encephalitides (limbic encephalitis, encephalitis lethargica) but also narcolepsy, or Guillain-Barré syndrome.

\section{Implications for neurological disorders COVID-19 infection in people with neurological diseases}

It is plausible that people with neurological disability and patients affected by diseases requiring immunomodulatory and immunosuppressive treatments may be at higher risk both of developing COVID-19, depending on personal and social circumstances influencing exposure, and of having a more severe form due to the underlying condition, weakened immunity, and disability status.

\section{Neuroimmunological disorders treated with immunosuppressive and immunomodulatory drugs Multiple sclerosis}

From the early days of the coronavirus pandemic, concerns emerged about the safety of the numerous people with MS, in particular those on immunomodulatory therapies, worldwide. In the absence of an evidence base, various neurological and patient organisations have attempted to produce guidelines that align to the general COVID-19 guidelines (e.g. Centres for Disease Control and Prevention: www.cdc.gov/coronavirus/2019-ncov/index.html) and balance the risk of exposure to the virus in patients on immunosuppressive or immunomodulatory therapy, with the risk of disease exacerbations in the face of discontinuation of treatment. The first such guidelines come from Italy, a country significantly affected by COVID-19 and with a substantial MS population. Many subsequent guidelines in other countries have been similar. ${ }^{51}$ In summary, patients on non-lymphodepleting therapies, such as first-line treatments (glatiramer acetate, interferon- $\beta$ ), dimethyl fumarate, teriflunomide and fingolimod, are generally advised in these guidelines to continue with the treatment; however, individual judgment is very important, as the latter three drugs have substantial immunosuppressive properties. Natalizumab treatment is also part of this category.

An additional rationale here is the risk of rebound disease activity and neuroinflammation on discontinuation (seen also with fingolimod). Most centres have also reduced the frequency of administration of natalizumab to extended dose interval (every 6-8 weeks), which has shown similar efficacy and significantly lower risk of progressive multifocal leukoencephalopathy. In patients on lymphoid-depleting therapies such as alemtuzumab, cladribine, ocrelizumab and rituximab, the advice is to delay the maintenance dose interval for those who have already been initiated, and to postpone the first dose in those who have not started yet. Some theoretical differences between treatments in this category have been brought up, and the argument that a more nuanced approach, such as using drugs that partially spare the antiviral response has been made. ${ }^{52}$ Rituximab treatment is already guided by B-cell count reconstitution in some conditions (e.g., B-cell proportion of $1 \%$ for neuromyelitis optica, to prevent secondary antibody deficiency), ${ }^{53}$ and this is likely to be implemented more widely, including in MS and ocrelizumab treatment.

Reassuringly, a recent Italian series reported only 232 patients with MS that had suspected or confirmed COVID-19 infection. Of these, $96 \%$ had mild disease, regardless of their treatment, and only 10 had severe or critical disease. All patients dying of COVID-19 had progressive MS and only two of these were on disease-modifying treatment (dimethyl fumarate and rituximab). These data, obtained from a country with a very large number of COVID-19 cases and fatalities, indicate that immunosuppressive or immunomodulatory treatment may not pose enormous risks. However, caution is still absolutely necessary with these groups of patients.

For patients with MS developing COVID-19 infection while on disease-modifying treatment, the guidelines recommend discontinuing treatment, with the possible, optional, exception of interferon- $\beta$, which, due to its antiviral properties, is currently in clinical trials for COVID-19. ${ }^{.54}$

\section{Myasthenia gravis, neuromyelitis optica spectrum disorders and immune-mediated neuropathies}

similar principles inform the guidelines for the above conditions.5.50 The balance between the risk of undertreating the neurological condition and the risk of infection needs to be assessed, in particular for conditions known to be typically more severe, such as neuromyelitis optica spectrum disorders. The risk of non-treatment, in particular in myasthenia gravis, dictates an approach whereby continuation of immunosuppressive treatment is encouraged. Generally, the risk is higher if immunosuppressive treatments with mycophenolate, 
azathioprine or rituximab are combined with prednisolone treatment over $20 \mathrm{mg} /$ day. Intravenous immunoglobulins or plasma exchange are generally reserved for acute exacerbations of specific conditions such as myasthenia gravis. Maintenance therapy probably needs to continue in patients receiving it, but this is not conducive to the rules of social distancing. Patients with myasthenia gravis are particularly at risk given that their neuromuscular weakness can further compromise their respiratory function in the setting of COVID-19. There are anecdotal reports of patients with myasthenia gravis developing COVID-19 and recovering well, and other (mainly elderly and with comorbidities) developing serious or fatal infection, but the full extent of the impact on patients with these diseases is not yet known.

\section{Other neurological conditions not treated with immunosuppressive or immunomodulatory agents Muscle disease and neuromuscular disease}

Patients with congenital muscle disease and neuromuscular diseases, such as amyotrophic lateral sclerosis, are at high risk of COVID-19 complications due to their reduced respiratory capacity and impaired cough reflex, and typically need to self-isolate during the coronavirus pandemic. The period of self-isolation will likely need to exceed the period of social distancing in the general population. Patients with cardiac muscle involvement on treatment need to continue their current cardiac treatment including ACE inhibitors, as there is no evidence to support a detrimental effect of these drugs on COVID-19 outcome. .5

\section{Stroke}

Patients with stroke are considered to be at higher risk of coronavirus complications. They are often in older age groups and have additional risk factors for COVID-19 complications, such as hypertension and diabetes.

\section{Other neurodegenerative diseases and dementia}

Patients with neurodegenerative diseases, like Parkinson's disease, Huntington's disease, Alzheimer's disease and related dementias, in particular those who live in care homes, represent an especially vulnerable group. Cognitive impairment, frailty, comorbidity and barriers to social distancing all contribute to the susceptibility and risk of complications of COVID-19. There are several reports of outbreaks affecting multiple residents of the same care home, a phenomenon of a magnitude that has not yet been fully assessed, given the variability in the rates of testing people outside hospital settings in some countries.

\section{Conclusions}

COVID-19, caused by a novel coronavirus, SARS-CoV-2, has already changed society in unprecedented ways. As our knowledge improves, so will our ability to diagnose and deal with neurological problems associated with this infection. COVID-19 poses huge challenges, but there are also opportunities. Guidelines are being produced at fast rates, and are subject to frequent updates as knowledge grows. Registers of affected patients are being created, and collaborations across disciplines and centres are forged. The Italian database is a collaboration of epidemiologists and neurologists. In the UK, existing registers (e.g., the UK MS Register) now capture COVID-19 information from participants.

Our neurological practice has changed and some of the changes may persist after the pandemic. Increased data sharing and the widespread introduction of telemedicine are more general examples. ${ }^{57}$ For those of us who deal with MS on a daily basis, more specific examples may be the adoption of extended dosing interval for many patients on natalizumab, of flexible interval for ocrelizumab, and, maybe, an increased use of interferon- $\beta$ ? We also need renewed energy in tackling comorbidities, actively promoting smoking cessation programmes, and reducing obesity and insulin resistance in our patients. •
1. Johns Hopkins University. COVID-19 Dashboard by the Center for Systems Science and Engineering (CSSE) at Johns Hopkins University (JHU). 2020. Available at: https://coronavirus.jhu.edu/ map.html (accessed 9 June 2020).

2. Corman VM, Muth D, Niemeyer D, Drosten C. Hosts and sources of endemic human coronaviruses. Adv Virus Res. sources of endem

3. Holmes KV. Coronaviruses and their replication. In Fundamental Virology 2 nd edition. New York: Raven Press, 1990;471-86.

4. Rehman SU, Shafique L, Ihsan A, Liu Q. Evolutionary trajectory for the emergence of novel coronavirus SARS-COV-2. Pathogens. 2020;9:240.

5. Wu F, Zhao $S$, Yu B, et al. A new coronavirus associated with human respiratory disease in China. Nature. 2020;579:265-9.

6u B, Zeng $L P$, Yang $X L$, et al. Discovery of a rich gene pool of bat SARS-related coronaviruses provides new insights into the origin of SARS Coronavirus. PLOS Pathog 2017:13.01006698. Origh O SARS COFOnavins. PLOS Pathog. 2017,13.e 1006698. Zou $x$, Chen $x$, Zou J, et al. Single-cell RNA-seq data analysis different human organs vulnerable to 2019-nCoV infection. different human organs vul
Front Med. 2020:14:185-92.

8. Baig AM, Khaleeq A, Ali U, Syeda H. Evidence of the COVID-19 virus targeting the cns: tissue distribution, host-virus interaction, and proposed neurotropic mechanisms. ACS Chem Neurosci. 2020;11:995-8.

9. Hamming I, Timens W, Bulthuis ML, et al. Tissue distribution of ACE2 protein, the functional receptor for SARS coronavirus. A first step in understanding SARS pathogenesis. J Pathol. 2004;203:631-7.

10. Sakima A, Averill DB, Gallagher PE, et al. Impaired heart rate baroreflex in older rats: role of endogenous angiotensin-(1-7) at the nucleus tractus solitarii. Hypertension. 2005;46:333-40.

11. Deng $Y$, Tan $X$, Li ML, et al. Angiotensin-converting enzyme 2 in the rostral ventrolateral medulla regulates cholinergic signaling the rostral ventrolateral medulla regulates cholinergic signaling rats. Neurosci Bull. 2019:35:67-78.

12. Brielle ES, Schneiderman-Duhovny D, Linial M. The SARS-Cov-2 exerts a distinctive strategy for interacting with the ACE2 human receptor. Viruses. 2020;12:487.

13. Raizada MK, Ferreira AJ. ACE2: a new target for cardiovascular disease therapeutics. I Cardiovasc Pharmacol. 2007;50:112-9.

14. Ulrich H, Pillat M. CD147 as a target for COVID-19 treatment: suggested effects of azithromycin and stem cell engagement. Stem Cell Rev Rep. 2020;16:434-40.

15. Kaushik DK, Hahn JN, Young WW. EMMPRIN, an upstream regulator of MMPs, in CNS biology. Matrix Biol.
2015;44-46:138-46.

16. Lavi E. Histopathology in coronavirus-induced demyelination. In: Lavi E, Constantinescu CS. Experimental models of multiple In: Lavi E, Constantinescu CS. Experimental
sclerosis. New York: Springer, 2005;711-6.

17. Li Y, Fu L, Gonzales DM, Lavi E. Coronavirus neurovirulence correlates with the ability of the virus to induce proinflammatory cytokine signals from astrocytes and microglia. J Virol. 2004;78:3398-406.

18. Lavi E, Fishman PS, Highkin MK, Weiss SR. Limbic encephalitis after inhalation of a murine coronavirus. Lab Invest. 1988;58:31-6.

9. Mahy BW. Molecular biology of the coronaviruses. Nature. 1983;305:474-5.

20. Burks JS, DeVald BL, Jankovsky LD, Gerdes JC. Two coronaviruses isolated from central nervous system tissue of two multiple sclerosis patients. Science. 1980;209:933-4.

21. Moriguchi T, Hairi N, Goto J, et al. A first case of meningitis/encephalitis with SARS-coronavirus-2 Int J Infect Dis. 2020;94:55-8.

22. Li H, Liu SM, Yu XH, et al. Coronavirus disease 2019 (COVID-19): current status and future perspectives. Int I Antimicrob Agents. 2020;55:10595

23. Chen T, Dai Z, Mo P, et al. Clinical characteristics and outcomes of older patients with coronavirus disease 2019 (COVID-19) in Wuhan, China (2019): a single-centered, retrospective study. J Gerontol A Biol Sci Med Sci. 2020;75:1788-95.

24. WU D, Wu T, Liu Q, Yang Z. The SARS-CoV-2 outbreak: what we know. Int I Infect Dis. 2020;94:44-8.

25. Cascella M, Rajnik M, Cuomo A, et al. Features, evaluation and treatment coronavirus (COVID-19). In: StatPearls. Treasure Island (FL): StatPearls Publishing; 2020. Available at: www.ncbi.nlm. nih.gov/books/NBK554776/ (accessed 3 September 2020).

26. Casas GC, Catala A, Cerretero Hernandez G, et al. Classification of cutaneous manifestations of COVID-19: a rapid, prospective nationwide consensus study in Spain with 375 cases. nationwide consensus study
Br J Dermatol. 2020;183:3-4.

27. de Masson A, Bouaziz JD, Sulimovic L, et al. Chilblains are common cutaneous finding during the COVID-19 pandemic: a retrospective nationwide study from France. J Am Acad Derm. 2020;83:667-70.

28. Rodriguez-Morales AJ, Cardona-Ospina JA, Gutierrez-Ocampo $E$, et al. Clinical, laboratory and imaging features of COVID-19: a systematic review and meta-analysis. Travel Med Infect Dis. 2020;34:101623.

29. Guan WJ, Liang WH, Zhao Y, et al. Comorbidity and its impact on 1590 patients with Covid-19 in China: a nationwide analysis. Eur Respir J. 2020;55:2000547.
30. Cui S, Chen S, Li X, et al. Prevalence of venous thromboembolism in patients with severe novel coronavirus pneumonia. J Thromb Haemost. 2020:18:1421-4.

31. Chen $T$, Wu D, Chen $\mathrm{H}$, et al. Clinical characteristics of 113 deceased patients with coronavirus disease 2019: retrospective study BMI 2020:368:m1091.

32. Wang L, He W, Yu X, et al. Coronavirus disease 2019 in elderly patients: characteristics and prognostic factors based on 4-week follow-up. I Infect. 2020;80:639-45.

33. Grasselli G, Zangrillo A, Zanella A, et al. Baseline characteristics and outcomes of 1591 patients infected with SARS-COV-2 admitted to ICUs of the Lombardy region, Italy. JAMA. 2020;323:1574-81.

34. Barrasa H, Rello J, Tejada S, et al. SARS-Cov-2 in Spanish intensive care: early experience with 15 -day survival in Vitoria. Anaesth Crit Care Pain Med. 2020;39:553-61.

35. Ferner RE, Aronson JK. Chloroquine and hydroxychloroquine in COVID-19. BMJ. 2020;369:m1432.

36. Amirian ES, Levy JK. Current knowledge about the antivirals remdesivir (GS-5734) and GS-441524 as therapeutic options for coronaviruses. One Health. 2020;9:100128.

37. Barlow A, Landolf KM, Barlow B, et al. Review of emerging pharmacotherapy for the treatment of coronavirus disease 2019. Pharmacotherapy. 2020;40:416-37

38. Shen $\mathrm{C}$, Wang $\mathrm{Z}$, Zhao F, et al. Treatment of 5 critically ill patients with COVID-19 with convalescent plasma. JAMA. 2020;323:1582-9

39. Bloch EM, Shoham S, Casadevall A, et al. Deployment of convalescent plasma for the prevention and treatment of COVID-19. J Clin Invest. 2020;130:2757-65.

40. Geleris J, Sun Y, Platt J, et al. Observational study of hydroxychloroquine in hospitalized patients with COVID-19. New Engl J Med. 2020;382:2411-8.

41. Yun W, Mao $C$, Lun $X$, et al. Structural basis for inhibition of the RNA-dependent RNA polymerase from SARS-COV-2 by remdesivir. Science. 2020;368:1499-504.

42. Mao $L$, Jin $H$, Wang $M$, et al. Neurologic manifestations of hospitalized patients with coronavirus disease 2019 in Wuhan, China. JAMA Neurol. 2020;77:683-90.

43. Lechien JR, Chiesa-Estomba CM, De Siati DR, et al. Olfactory and gustatory dysfunctions as a clinical presentation of mild-to-moderate forms of the coronavirus disease (COVID-19): a multicenter European study. Eur Arch Otorhinolaryngol. 2020;277:2251-61.

44. Helms J, Kremer S, Merdji H, et al. Neurologic features in severe SARS-CoV-2 infection. New Engl I Med. 2020;382:2268-70. 
45. Dolnikhof M, Duarte-Neto AN, de Almeida Monteiro RA, et al. Pathological evidence of pulmonary thrombotic phenomena in severe COVID-19. J Throm Haemost. 2020;18:1517-9.

Zhang $\mathrm{Y}$, Xiao $\mathrm{M}$, Zhang $\mathrm{S}$, et al. Coagulopathy and antiphospholipid antibodies in patients with COVID-19. N Eng/ J Med. 2020;382:e38.

47. Dafer RM, Osteraas ND, Biller J. Acute stroke care in the coronavirus disease 2019 pandemic. I Stroke Cerebrovasc Dis. 2020;29:104881

48. Poyiadji N, Shahin G, Noujaim D, et al. COVID-19-associated acute hemorrhagic necrotizing encephalopathy: $\mathrm{CT}$ and MR features. Radiology. 2020;296:E119-20.

49. Zhao H, Shen D, Zhou H, et al. Guillain-Barre syndrome associated with SARS-COV-2 infection: causality or coincidence? Lancet Neurol. 2020;19:383-4.

50. Sormani MP, Italian Study Group on COVID-19 infection in multiple sclerosis. An Italian programme for COVID-19 infection in multiple sclerosis. Lancet Neurol. 2020:19:481-2.

51. Giovannoni G, Hawkes C, Lechner-Scott, et al. The CoviD-19 pandemic and the use of MS disease-modifying therapies.

52. Amor S, Baker D, Khoury S, et al. SARS-CoV-2 and multiple sclerosis: not all immune depleting DMTs are equal or bad Ann Neurol. 2020;87:794-7.

53. Tallantyre EC, Whittam DH, Jolles S, et al. Secondary antibody deficiency: a complication of anti-CD20 therapy for neuroinflammation. J Neurol. 2018;265:1115-22.

54. Sallard E, Lescure FX, Yazdanpanah Y, et al. Type 1 interferons as a potential treatment against COVID-19. Antiviral Res. 2020;178:104791.

55. Guidon AC Amato AA COVID-19 and neuromuscular disorders. Neurology. 2020;94:959-69.

56. Brownlee W, Bourdette D, Broadley S, et al. Treating multiple sclerosis and neuromyelitis optica spectrum disorder during the COVID-19 pandemic. Neurology. 2020;94:949-52

57. Klein BC, Busis NA. COVID-19 is catalyzing the adoption of teleneurology. Neurology. 2020;94:903-4. 